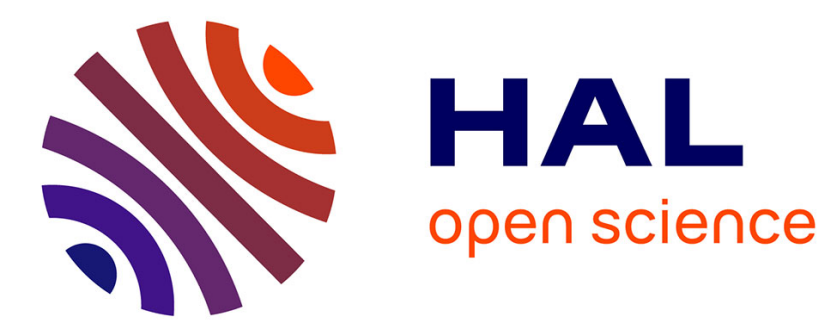

\title{
ULF waves in the Io torus: ULYSSES observations
} Naiguo Lin, P. J. Kellogg, R. J. Macdowell, Y. Mei, N. Cornilleau-Wehrlin, P. Canu, C. de Villedary, L. Rezeau, A. Balogh, R. J. Forsyth

\section{To cite this version:}

Naiguo Lin, P. J. Kellogg, R. J. Macdowell, Y. Mei, N. Cornilleau-Wehrlin, et al.. ULF waves in the Io torus: ULYSSES observations. Journal of Geophysical Research Space Physics, 1993, 98 (A12), pp.21151-21162. 10.1029/93JA02593 . hal-00408544

\section{HAL Id: hal-00408544 https://hal.science/hal-00408544}

Submitted on 3 Feb 2021

HAL is a multi-disciplinary open access archive for the deposit and dissemination of scientific research documents, whether they are published or not. The documents may come from teaching and research institutions in France or abroad, or from public or private research centers.
L'archive ouverte pluridisciplinaire HAL, est destinée au dépôt et à la diffusion de documents scientifiques de niveau recherche, publiés ou non, émanant des établissements d'enseignement et de recherche français ou étrangers, des laboratoires publics ou privés. 


\title{
ULF Waves in the Io Torus: Ulysses Observations
}

\author{
Naiguo Lin, ${ }^{1}$ P. J. Kellogg, ${ }^{1}$ R. J. MacDowall, ${ }^{2}$ Y. Mei, ${ }^{3}$ N. Cornilleau-Wehrlin, ${ }^{4}$ \\ P. CANU, ${ }^{4}$ C. DE VILledary, ${ }^{4}$ L. RezeaU, ${ }^{4}$ A. Balogh, ${ }^{5}$ AND R. J. FORSYTH ${ }^{5}$
}

\begin{abstract}
Throughout the Io torus, Ulysses has observed intense ULF wave activity in both electric and magnetic components. Such ULF waves have been previously suggested as the source of ion precipitation leading to Jovian aurorae. The peaks of the wave spectra are closely related to the ion cyclotron frequencies, which is evidence of the waves being ion cyclotron waves (ICWs). Analysis of the dispersion relation using a multicomponent density model shows that at high latitudes $\left(\sim 30^{\circ}\right)$, peak frequencies of the waves fall into $L$ mode branches of guided or unguided ICWs. Near the equator, in addition to the ICWs below $f_{\mathrm{cO}}++$, there are strong signals at $\sim 10 \mathrm{Hs}$ which require an unexpectedly large energetic ion temperature anisotropy to be explained by the excitation of either convective or nonconvective ion cyclotron instabilities. Their generation mechanism remains open for the future study. Evaluation of the Poynting vector and the dispersion relation analysis suggest that the waves near the equator had a small wave angle relative to the magnetic field, while those observed at high latitudes were more oblique. The polarization of the waves below $f_{c H^{+}}$is more random than that of the whistler mode waves, but left-hand-polarised components of the waves can still be seen. The intensity of the ICWs both near the equator and at high latitudes are strong enough to meet the requirement for producing strong pitch angle scattering of energetic ions.
\end{abstract}

\section{INTRODUCTION}

The ultralow frequency (ULF) waves of interest here are the waves near and below the local ion cyclotron frequencies. The existence of these waves in the Jovian magnetosphere has received attention [e.g., Thorne and Moses, 1983, 1985; Gurnett and Goertz, 1983; Mei et al., 1992] because it may account for the depletion of energetic particles in the Io torus [Krimigis et al., $1979]$ and the excitation of extreme ultraviolet emissions observed by Voyager 1 [Broadfoot et al., 1979]. Such low-frequency waves, most likely ion cyclotron waves (ICWs), could be the waves required to scatter energetic ions into the loss cone at the strong pitch angle diffusion limit and cause strong ion precipitation into the auroral zone. Thorne and Moses [1983, 1985] noticed that the ICWs with a frequency above the orygen gyrofrequency could be excited strongly at high latitudes $\left(>15^{\circ}\right)$ and would be damped by ion cyclotron damping when propagating toward the equator.

More recently, Mei et al. [1992], using realistic plasma parameters, have given a complete evaluation of convec-

\footnotetext{
${ }^{1}$ School of Physics and Astronomy, University of Minnesota, Minneapolis.

2NASA Goddard Space Flight Center, Greenbelt, Maryland.

${ }^{3}$ Department of Atmospheric Sciences, University of California, Los Angeles.

${ }^{4}$ Centre de Recherche en Physique de l'Environnement, Centre National d'Etudes des Télécommunications, Issy les Moulineaux, France.

${ }^{5}$ Imperial College of Science and Technology, London, United Kingdom.
}

Copyright 1993 by the American Geophysical Union.

Paper number 93JA02593.

0148-0227/93/93JA-02593\$05.00 tive growth of ICWs at all frequencies below the proton gyrofrequency in the entire Io plasma torus by wave ray tracing. They found that there are preferred regions of instability for the ICWs at different frequencies in the Io torus: (1) The waves between the proton and oxygen gyrofrequencies $(\sim 10 \mathrm{~Hz})$ grow only marginally and only at higher magnetic latitudes $\left(\Lambda>15^{\circ}\right)$. They are confined to magnetic shells near $L=8$. The path-integrated gain of these waves is very sensitive to the plasma parameters. (2) The waves with lower frequencies (below the $\mathrm{O}^{+}$gyrofrequency, $f_{c \mathrm{O}^{+}}$) may experience strong growth at the equator. They can propagate to high-latitude regions without damping until absorbed by thermal heavy ions.

While the ion cyclotron instability in the Earth's magnetosphere has long been observed and widely studied as Pc 1 and 2 micropulsations (see, for example, Kennel and Petschek [1966], Mauk et al. [1981], Fraser [1982], and Roux et al. [1982]), there have been only a few observations of Jovian ULF waves. Thorne and Scarf [1984] reported an enhancement in wave activity observed by Voyager 1 while traversing the Io torus, and the wave intensification was strongest in the frequency range below the proton gyrofrequency. Although the observed average wave intensity was typically an order of magnitude smaller than the strong pitch angle diffusion limit, the authors interpreted the wave activity as evidence for the possible existence of strong ICW sources at higher latitudes which were not reached by the spacecraft.

During the Ulysses flyby of Jupiter on February 8 , 1992, the spacecraft passed through the Io torus from north to south traversing a large range of magnetic latitudes. It provided an opportunity to observe waves at high latitudes which had not been reached previously as well as those in the equatorial region. Furthermore, the waveform analyzer (WFA) of the unified radio and plasma wave instrument (URAP) onboard the Ulysses spacecraft measures both electric and magnetic signals 
with a wide frequency range from $\sim 500 \mathrm{~Hz}$ to a fraction of hertz, which is suitable for the study of the ULF wave we have reviewed above. It is the first spacecraft to carry a magnetic search coil into the Jovian magnetosphere. We will present the observations of ULF waves during the flyby and discuss their possible generation mechanisms.

\section{INSTRUMENTATION}

A detailed description of the URAP experiment has been presented by Stone et al. [1992a]. The data used in this study are derived from the WFA, which provides spectral analysis of electric and magnetic signals in 10 low-band and 12 high-band channels. The central frequencies of the low-band channels are $0.22,0.33,0.44$, $0.67,0.89,1.33,1.78,2.67,3.56$, and $5.33 \mathrm{~Hz}$, while those of the high band are 9.3, 14.0, 19.0, 28.0, 37.0, $56.0,75.0,112,149,224,299$, and $448 \mathrm{~Hz}$.

For the high-band channels, electric and magnetic field measurements on antennas which are perpendicular to the spin axis, are available. They are denoted here as $E_{x}$ and $B_{y}$, respectively. Thus $E_{x}$ and $B_{y}$ are measured in a rotating frame of reference and the $z$ and $y$ refer to rotating spacecraft coordinates. For the $z$ antennas, which are aligned with the spin axis and always point to the Earth, the electric and magnetic signals, $E_{z}$ and $B_{z}$, are measured alternatively. For the lowband channels, data are obtained for $E_{x}$ and either $B_{y}$ or $B_{z}$. In this paper the electric signals are converted to field strength $\left((\mathrm{V} / \mathrm{m})^{2} / \mathrm{Hz}\right)$ by dividing by an effective length of $35 \mathrm{~m}$ for the $z$ antennas and $3.5 \mathrm{~m}$ for the $z$ antenna.

In addition to the average data set for both high and low bands, which consists of the averages of available $E_{x}$, $E_{z}, B_{y}$, and $B_{z}$ over $64 \mathrm{~s}, \sim 5$ times the spin period (12 s), there are two more sets of data obtained in high-band channels: (1) a peak data set, which is the largest value recorded for the $E_{x}$ channels for each frequency and for each octant of spacecraft rotation, and the values of $B_{y}$ and $B_{z}$ or $E_{z}$ taken at the same time, and (2) a measurement of the phase difference between the $E_{x}$ signal and the two others at the same time that the peak measurement was made. The resolution of the phase measurements is $22.5^{\circ}$. This set of data may be used to study the polarization of the signals.

\section{ObSERVATIONS}

The trajectory of Ulysses during the flyby of Jupiter is shown in Figure 1. Contours of equal electron density are overplotted in Figure 1. The density numbers are derived from the equatorial plasma density (F. Bagenal, private communication, 1993) using a diffusive equilibrium model [Mei et al., 1992; Mei, 1992]. Between 0700 and 2000 UT of Feb. 8, Ulysses traveled from north to south covering magnetic latitudes between $\sim+48^{\circ}$ and $\sim-15^{\circ}$. It reached the closest point to Jupiter at $\sim 1202 \mathrm{UT}$ at a radial distance of $6.31 R_{J}$ at $0130 \mathrm{LT}$, then penetrated the Io plasma torus. At $\sim 1614$ UT the spacecraft crossed the magnetic equator at a distance of $\sim 7.75 R_{J}$ from Jupiter.

Dynamic spectra of electric and magnetic fields measured by the WFA during the day of February 8 are presented in Plate 1. The data displayed have been corrected for the background noise level of the instrument

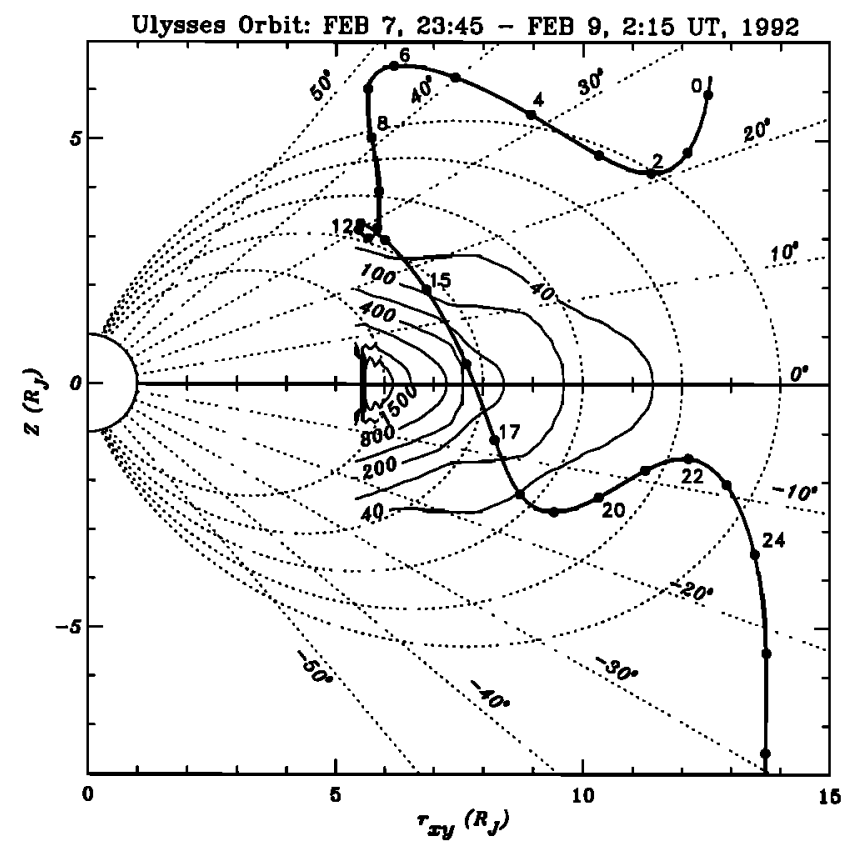

Fig. 1. The trajectory of Ulysses between 2345 UT, February 7, and 0215 UT, February 9, 1992, plotted in magnetic dipole field coordinates, where the horizontal axis ray is the cylindrical radius from the center of Jupiter, and the vertical axis is the cylindrical $x$ from Jupiter. The solid circles show the position of Ulysses for every hour from 0000 of February 8 to 0200 of February 9. The thin lines are contours of equal electron density (per cubic centimeter).

and are then divided by a polynomial fit of the background noise. The noise level is determined empirically from previous quiet periods and is displayed along with the fit in Figure 2, which shows examples of line plot spectra. We note that during the entire period of Io torus traversal, signals detected in all channels of WFA were always a few decades higher in intensity than the noise level, indicating that there are strong and active sources of various plasma waves in the lo torus.

The upper panel of Plate 1 shows such relative intensities of electric signals detected on $E_{x}$; while the lower panel shows the relative intensity of magnetic signals, $B_{y}$, (or, for some periods for the low-band channels, of $B_{z}$ ). Both electric and magnetic data are the 64-s averages. Local ion cyclotron frequency lines calculated using the Ulysses magnetometer data are overplotted on each panel.

Intense wave activity is seen in both the electric and magnetic data throughout the day. The waves near and above the proton gyrofrequency and extending to the upper limit of WFA frequency $(448 \mathrm{~Hz}$ ) are believed to be varieties of whistler mode waves. They are the topics of other studies and will only be mentioned here briefly. The wave enhancement between 0400 and 0800 UT has been suggested to be auroral hiss [Farrell et al., 1993] whose sources may be located in the boundary between open and closed Jovian magnetic field lines. Another intense period of wave activity at a few hundred hertz was seen between 1000 and 1500 UT. These waves, which are believed to be whistler mode chorus, were observed up to $2 \mathrm{kHz}$ by Ulysses [Stone et al., 1992b] and are dis- 
cussed extensively by Farrell et al. [this issue]. The above wave activity was seen in both electric and magnetic components, which confirmed observationally for the first time the electromagnetic nature of the waves.

Enhanced waves below $f_{\mathrm{CH}^{+}}+$were observed after 0800 when Ulysses was penetrating the Io plasma torus. Between 1000 and $1400 \mathrm{UT}$, two peaks of wave signals were observed in the $E_{x}$ component. One is probably right below the local $\mathrm{O}^{++}$gyrofrequency, and the other is below $f_{q^{+}} \cdot$. Note that the $E_{\approx z}$ signals of whistler mode hiss during this interval extended to $\sim 10 \mathrm{~Hz}$, below the $f_{\mathrm{cH}}+$ line and partially obscure the first peak. The $B_{y}$ spectrum also shows similar enhancement below $f_{\mathrm{cH}^{+}}$, but the second peak below $f_{\mathrm{cs}}+$ does not appear in this spectrum. We note that during this 4-hour interval the spacecraft was essentially staying in the same $L$ shells $(L \sim 8.5)$ at about the same magnetic latitude $\left(\sim 30^{\circ}\right)$.

After 1400 , as the spacecraft went south approaching the magnetic equator, the intensity of $E_{*}$ gradually decreased and reached its minimum at the equator, then increased again gradually as the spacecraft went further southward. The sudden decrease at 1910 in low-band frequencies in $E_{x}$ was due to a gain change of preamplifiers which saturated the electronic system. Despite the saturation, we still see that the intensity after the equator crossing was not as strong as that before the crossing. The time variation of the $B_{y}$ spectra near the equator appears to be different from that of $E_{z}$. The $B_{y}$ component has a strong enhancement near the equator and peaks at the channel with the central frequency right below the local proton frequency. We will see later that the apparent difference in $E_{z}$ and $B_{y}$ spectra at the equator may be caused by the change in direction of the observations of the wave front plane.

In Figure 2 we show line spectra of two periods to be examined in more detail: the period between 1155 and 1210, when the spacecraft was at $L \sim 8.5$, and magnetic latitudes of $\sim 30^{\circ}$ (Figure 2a), and the period between 1605 and 1620 , when the spacecraft was near the magnetic equator at $L \sim 7.7$ (Figure $2 b$ ). In these two periods, measurements of $B_{2}$ were made for the high-band channels only. The background noise of the instrument is plotted in the lower panels for each component. Figures $2 a$ and $2 b$ show that the power density in all components are at least one or two decades higher than the instrument noise.

We marked with arrows the local ion cyclotron frequencies, $f_{\mathrm{cH}^{+}}, f_{\mathrm{cO}}+, f_{\mathrm{cS}^{+}}$and $f_{\mathrm{co}++}$, in the upper panels of Figure 2, which display the relative intensity of each component to show the correspondence of features in the wave spectra to the cyclotron frequencies. For the high-latitude spectra (Figure $2 a$ ) a reduction in the wave intensity near the proton gyrofrequency $\left(f_{\mathrm{cH}^{+}} \sim 35.5 \mathrm{~Hz}\right.$ for the interval) can be seen in all three components. In $E_{x}$ this reduction is not as clear as in $B_{y}$ and $B_{z}$ probably because the $E_{x}$ signals of whistler mode hiss, which peak at $\sim 200-300 \mathrm{~Hz}$, extend to channels below $f_{\mathrm{cH}^{+}}$. In $B_{y}$ and $B_{z}$ the whistler mode signals showed a clear cutoff near $f_{\mathrm{cH}^{+}}$, and a relative marimum near $10 \mathrm{~Hz}$ may be seen in $B_{y}$ (and probably in $B_{z}$, too). In the $E_{x}$ spectra, another reduction in signal strength is seen near $f_{c \mathrm{O}^{+}}(\sim 2.2 \mathrm{~Hz})$ and another peak appears immediately below $f_{c}+$.
The above characteristics of the spectra seem to resemble the spectra of ion cyclotron waves observed previously in the Earth's magnetosphere. For example, Young et al. [1981] observed in $\mathrm{He}^{+}$-rich plasma the reduction in the wave intensity right above the helium gyrofrequency, which is believed to be in the stop band for ICWs between the $\mathrm{He}^{+}$gyrofrequency and a cutoff frequency. "Spectral slots" due to the stop band near the $\mathrm{O}^{+}$gyrofrequency have also been observed [cf. Fraser et al., 1992]. The similar spectral characteristics in Figure 2 suggest that the waves below $f_{\mathrm{cH}^{+}}$are associated with the ion cyclotron instability.

The spectra of equatorial waves (Figure $2 b$ ) show similar features. A peak at the frequency below $f_{c H}+(\sim 11.3$ $H_{z}$ ) appears in the $B_{y}$ component (and probably in $B_{z}$ ). The peak in $E_{x}$ is not as clear as in the case of the last interval. A reduction in the wave intensity near the gyrofrequency of $\mathrm{O}^{++}$can be seen in the $E_{x}$ spectra as well as in $B_{y}$, followed by a peak in the lower frequency channels. This may be evidence of the presence of $\mathrm{O}^{++}$ in this equatorial region $(L \sim 7.7)$, which has been observed by Voyager 1 [Bagenal et al., 1992].

We note that the frequency resolution of WFA is rather low for this study. Within a stop band near an ion gyrofrequency there may be only one or two channels as will be shown later. Also, a very strong signal in a channel of a given frequency may leak into the neighbor channels resulting in spurious signals in these channels. The above shortcomings might have affected the observations of the stop bands in the spectra and made them less evident.

It is interesting to compare the power density of signals on the $z$ antennas with those on the $z$ or $y$ antennas for the two periods. For example, Figure $2 a$ shows that at $9.3 \mathrm{~Hz}$, the ratio of the power density $B_{y} / B_{z}$ is $\sim 2$, while near the equator (Figure $2 b$ ), $B_{z}$ is more than 5 times larger than $B_{y}$. In Figure $3 a$ we plot the comparison between $E_{x}$ and $E_{z}$ or between $B_{y}$ and $B_{z}$ (64-s average data) for the channel of $9.3 \mathrm{~Hz}$ from 0900 to 1900 UT. The variation of the ratio of wave amplitudes $E_{x} / E_{z}$ and $B_{y} / B_{z}$ (the third panel) seems to be continuous and consistent. Before 1400 , when the spacecraft was at high latitudes $\left(>25^{\circ}\right)$, and the ambient magnetic field was mostly in the $-z$ direction, the amplitude of signals in the $x-y$ plane were $\sim 2$ times stronger than those along $z$. As the spacecraft approached the equator, and the magnetic field became more and more in the north-south direction, the ratio reversed as the signals detected on $z$ antenne became stronger than the $z-y$ signals. After the equatorial crossing the ratio tends to reverse again. The above variation pattern was seen in the high-band channels below $56 \mathrm{~Hz}$. There is no simultaneous measurements of $E_{z}$ and $E_{z}$ or $B_{y}$ and $B_{2}$ for the low-band channels, but it is likely that the above pattern also exists in low-band channels.

The variation of the ratio of the wave intensities may be explained at least partially by the change in the orientation of the wave front plane. In Figure $3 b$ we show the peak values of $E_{x}$ (in $\mathrm{V}^{2} / \mathrm{Hz}$ ) and $B_{y}\left(\right.$ in $\mathrm{nT}^{2} / \mathrm{Hz}$ ) versus the number of sectors for three successive time intervals when the spacecraft was near the equator. Sector 0 begins at the position when the $+x$ antenna is closest to the Sun, and thus the center of the sector is $22.5^{\circ}$ 

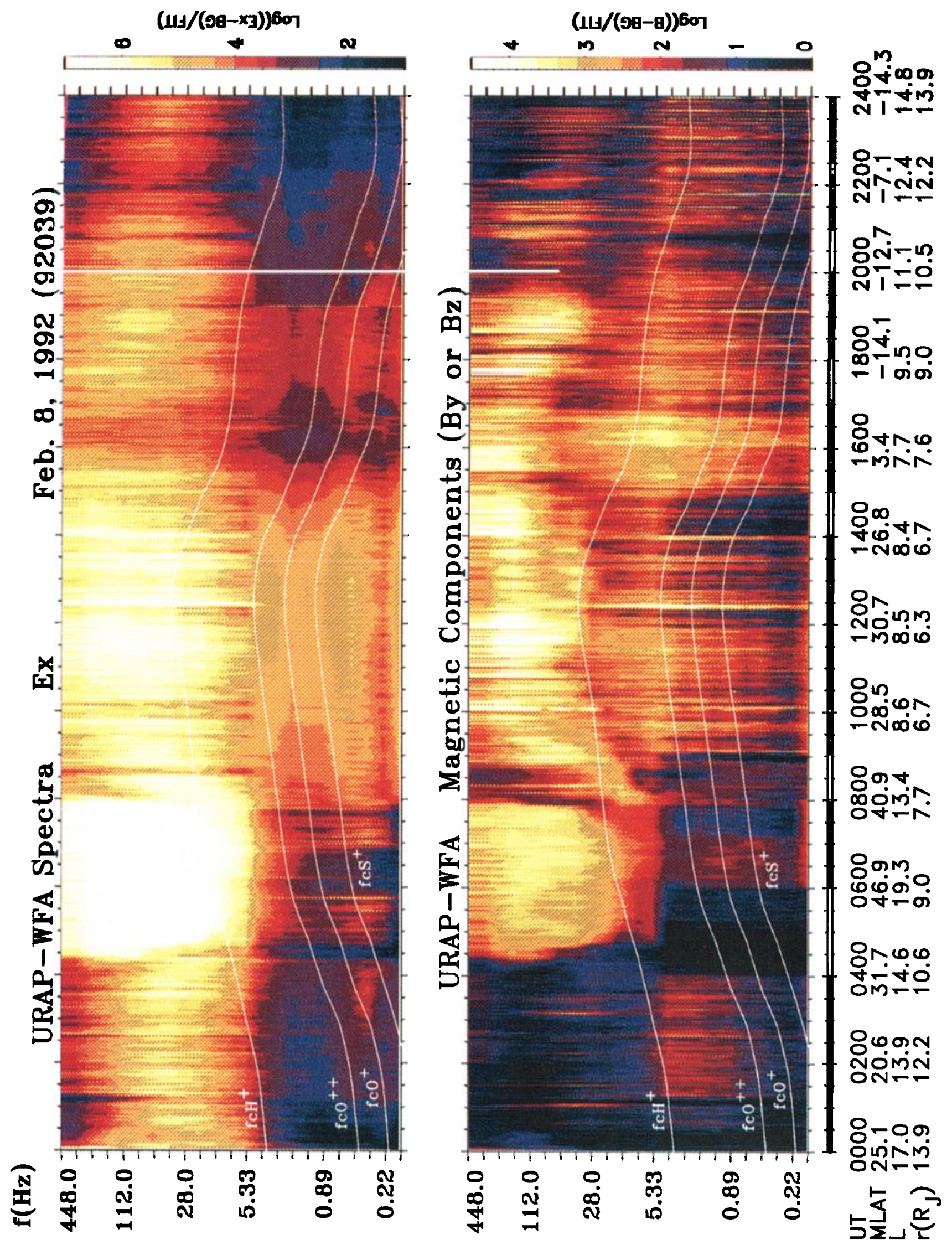

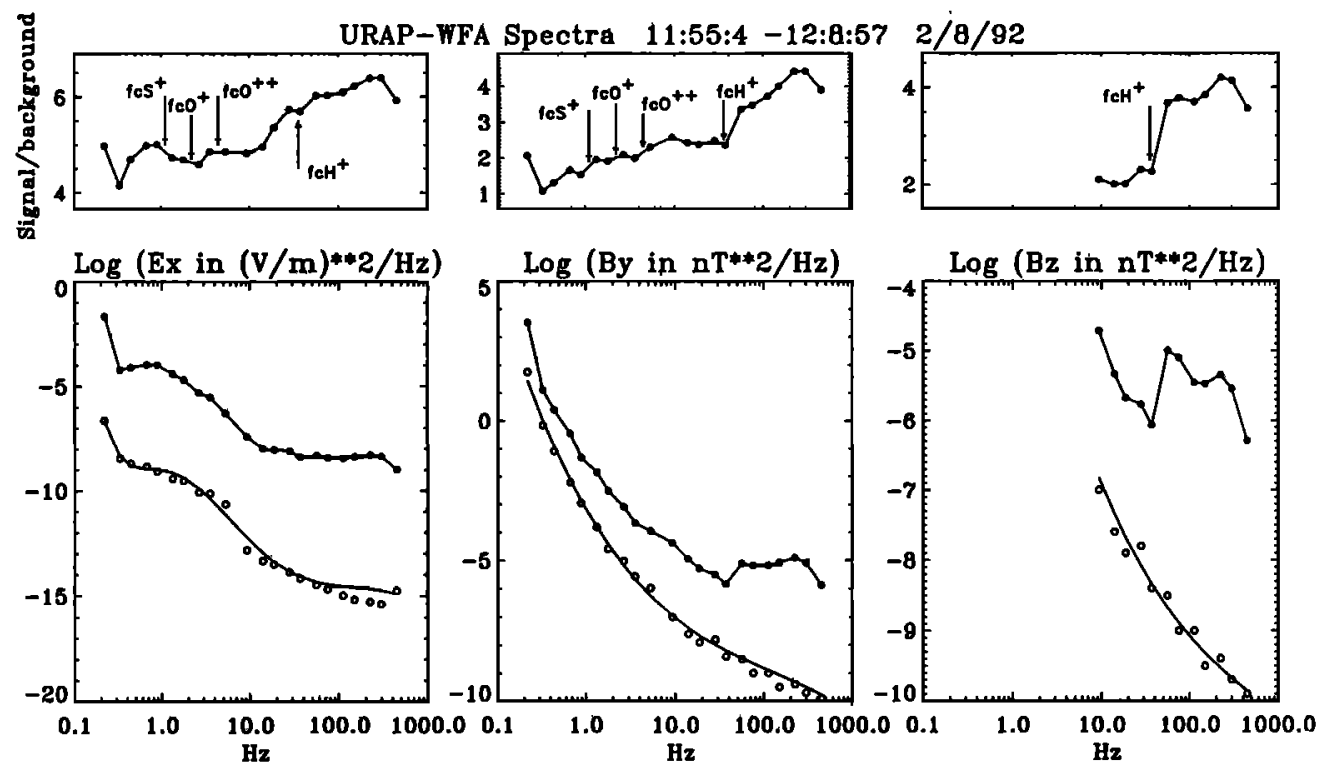

Fig. 2a. Spectra of (from left to right) $E_{z}, B_{y}$, and $B_{z}$ for the period 1155 to 1210 UT. The upper panel for each component is the logarithm of the relative intensity which is used to plot the dynamic spectra in Plate 1. In the lower panels the logarithm of the power density is shown. The instrument background noise is also plotted (open circles) for each component along with the polynomial fit to the noise. Arrows in the upper panels indicate the ion gyrofrequencies.

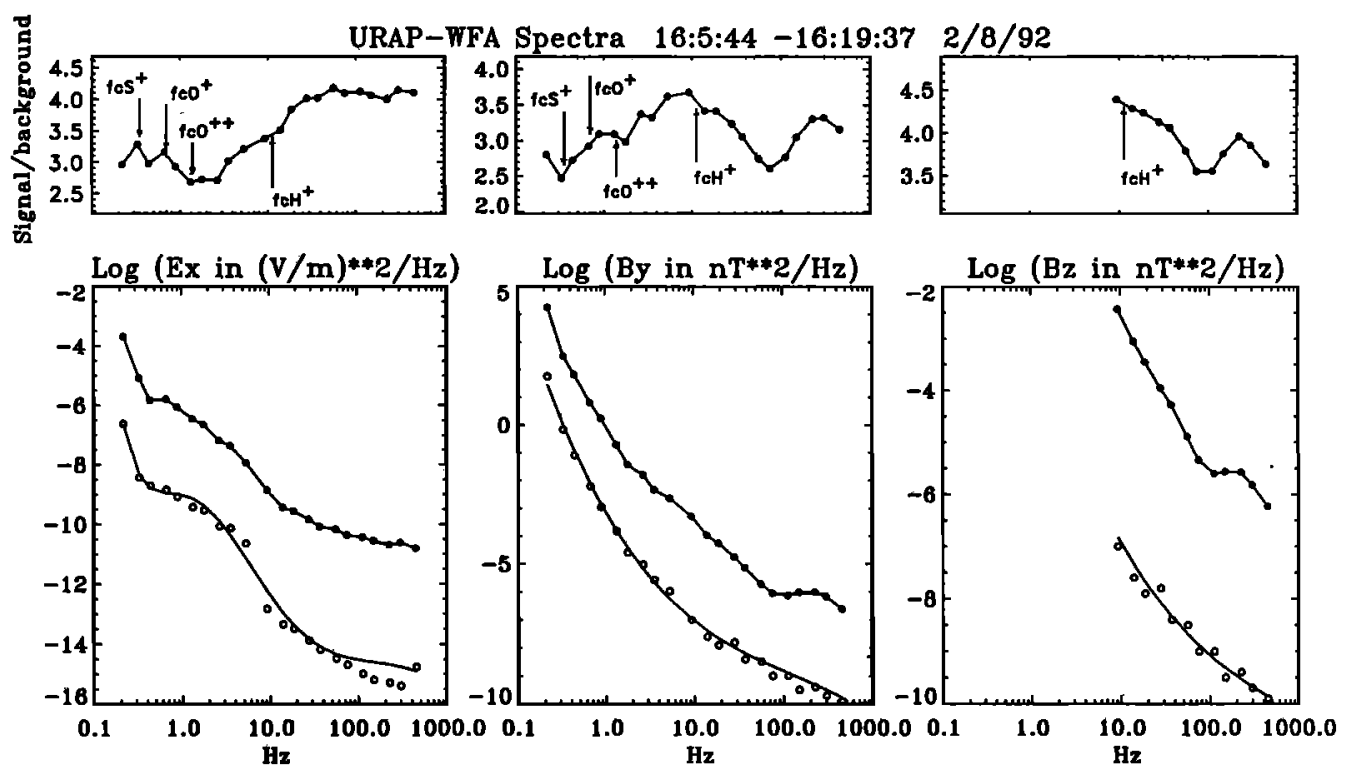

Fig. 2b. The same as Figure $2 a$ but for the period 1605 to 1620 UT.

Plate 1. Dynamic spectra of the relative intensity of (upper) $E_{\text {s }}$ and (lower) $B_{y}$. Each tickmark on the vertical ares indicates the center frequency of a waveform analyzer (WFÁ) channel. A horizontal bar under the second panel with alternative black and white periods indicates the intervals when the magnetic measurements in low-band channels were $B_{y}$ (black periods) or $B_{z}$ (white periods). For the high band (frequency $>5.3 \mathrm{~Hz}$ ) the displayed magnetic data are all $B_{y}$. The solid lines overplotted on each panel are local gyrofrequency lines for $\mathrm{H}^{+}, \mathrm{O}^{++}, \mathrm{O}^{+}$, and $\mathrm{s}^{+}$, labeled as $f_{c \mathrm{H}^{+}}, f_{\mathrm{cO}} \mathrm{O}_{+}, f_{\mathrm{cO}}$, and $f_{c S^{+}}$, respectively. The universal time (UT), magnetic latitude (MLAT), $L$ value, and the distance from Jupiter $r$ in $R_{J}$, are marked at the bottom of the figure. 


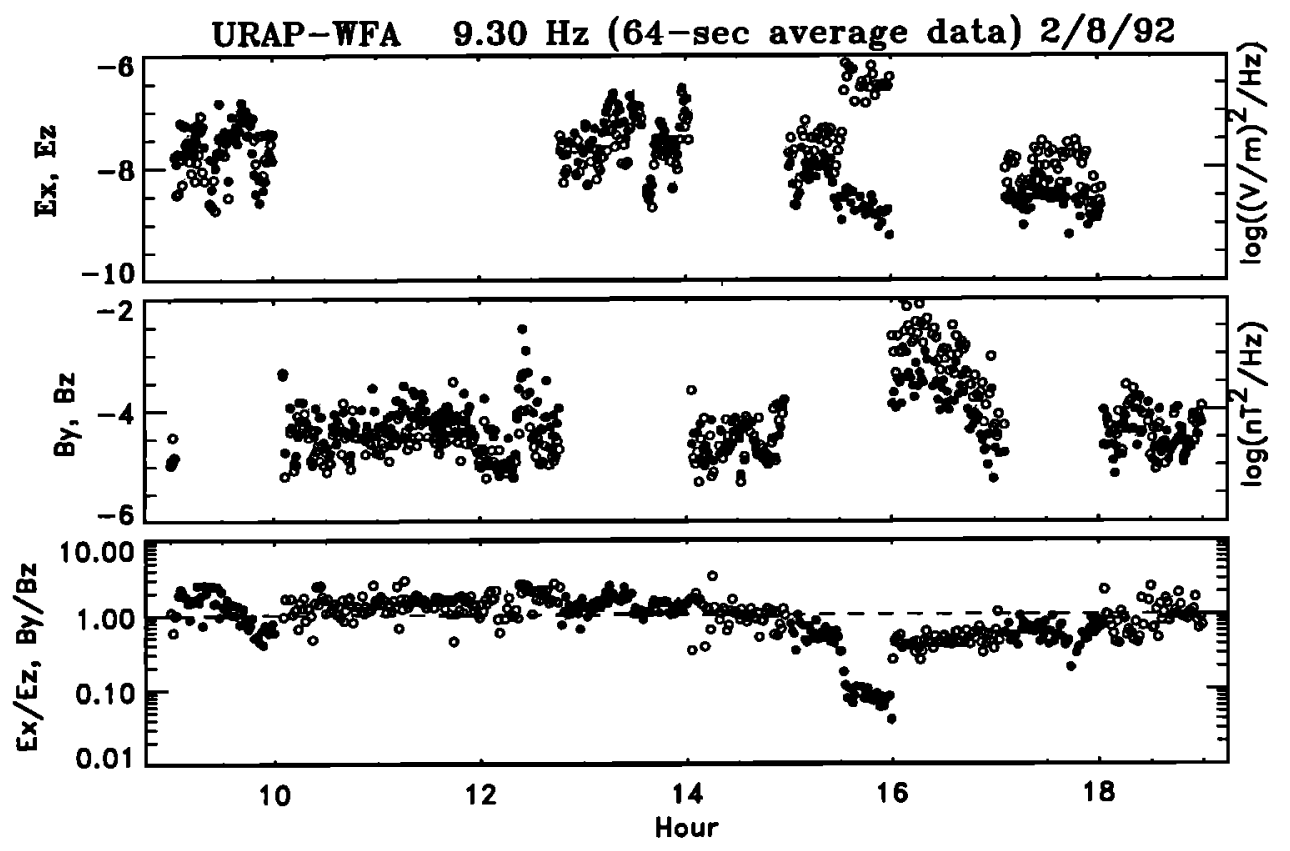

Fig. 3a. Comparison between $E_{x}$ and $E_{z}$, and $B_{y}$ and $B_{z}$ (64-s average data) for the 9.3-Hz channel from 0900 to 1900 UT. The first panel shows the logarithm of the power density of $E_{x}$ (dots) and $E_{z}$ (circles) when $E_{z}$ measurements are available. The second panel is $\log \left(B_{y}\right)$ (dots) and $\log \left(B_{z}\right)$ (circles). The bottom panel is the ratios of the wave amplitudes $E_{x} / E_{z}$ (dots) and $B_{y} / B_{z}$ (circles) plotted in log scale.

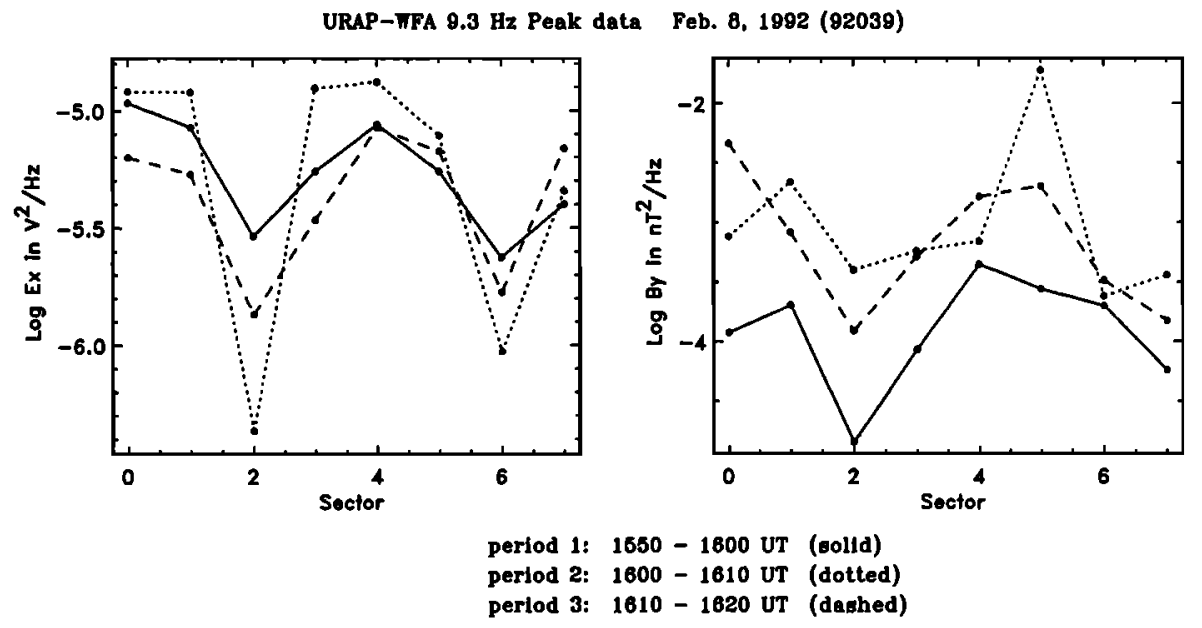

Fig. 36 . The logarithm of the peak values of (left) $E_{x}$ and (right) $B_{y}$ versus the number of sectors where the measurements of the peak data are taken for three periods near the equator: 1550-1600 UT (solid lines), 1600-1610 UT (dotted lines), and 1610-1620 UT (dashed lines).

away from the $+X$ axis of the despun spacecraft coordinates. During the period we are studying $+X$ pointed eastward and $+Y$ pointed southward. The $z$ antenna always coincides with the $Z$ axis. The relative position between the despun coordinates, the $x-y$ antennas, and the sector directions is illustrated in Figure 4.

Figure $\mathbf{3} b$ shows that the electric wave power is strongest in sectors 0 and 4 which are near the despun $X$ axis and has minima in sectors 2 and 6 near the $Y$ axis. The magnetic wave power has similar modulation but the maxima shift to sectors 1 and 5 in some periods. It seems that the waves observed may have their wave front roughly in a plane between $0-4$ and $1-5$ sectors and containing the $Z$ axis. The wave electric field and magnetic field lie in this plane have larger components in the $Z$ and in the $X$ directions than those in the $Y$ direction. Since $E_{x}$ and $B_{y}$ used in Figure $4 a$ are the $64 \mathrm{sec}$ (five spins) average of the measurements made in the $X-Y$ plane, while $E_{z}$ and $B_{z}$ are the average of the measurements on $z$ antenna only, thus $E_{z}$ and $B_{z}$ should be larger than $E_{x}$ and $B_{y}$. This is what we see near the equator ( 1600 UT) in Figure $3 a$. We note, however, while the ratio of the magnetic wave amplitudes $B_{y} / B_{z}$ is $\sim 0.5$ (see the third panel of Figure $3 a$ 


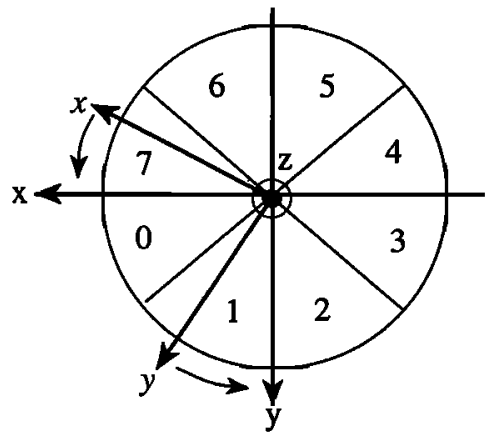

Fig. 4. The relative position between the despun spacecraft coordinates $(X, Y$, and $Z)$, the antennas $x, y$, and $z$, and the directions of the eight sectors as viewed from the Earth. The $+Z$ antenna points to the Earth, $+X$ lies in the plane containing the $+Z$ and the Sun, pointing toward the direction closest to the Sun and perpendicular to the $+Z$, and $+Y$ completes the orthogonal system. The antenna rotates in the direction indicated by the curved arrows. The $z$ antenna coincides with the $Z$ axis.

right after 1600), which seems reasonable, the ratio of the electric wave amplitudes $E_{x} / E_{z}$ (right before 1600) is $\sim 0.1$, which is unexpectedly small. One possible reason we can think of for such small $E_{x} / E_{z}$ is that the waves burst observed near the equator may also have a large electrostatic component in the $Z$ direction.

At higher latitudes the wave front seems to lie on a plane closer to the $X-Y$ plane and to be roughly perpendicular to the $Z$ axis, so that the wave components projected onto the $X-Y$ plane are larger than that onto the $Z$ direction. The changes in the direction of the wave front planes observed at different latitudes may explain the apparent decrease of $E_{x}$ and enhancement of $B_{y}$ toward the equator seen in Plate 1.

The observed wave intensity in $E_{z}$ at $9.3 \mathrm{~Hz}$ near the equator was $\sim 10^{-6}(\mathrm{~V} / \mathrm{m})^{2} / \mathrm{Hz}$. It is comparable to the peak wave intensity observed by Voyager 1 at $10 \mathrm{~Hz}$ at $L=6.2$ [Thorne and Scarf, 1984].

\section{ANalysis}

\section{Poynting Vector Evaluation}

In the high-band channels the WFA measures peak values of $E_{x}$ in each of the eight sectors in a spin rotation, and at the same time, the measurements of $B_{y}$, and $E_{z}$ or $B_{z}$ are made. The phases of the components with respect to $E_{x}$ are also measured for each sector.

We used the peak and phase data to evaluate the Poynting flux of the observed waves in the spacecraft coordinates. Since we do not have simultaneous measurements of all six components of $B$ and $\mathrm{E}\left(E_{z}\right.$ and $B_{z}$ are measured alternately), the calculations were made only in intervals (we took $10 \mathrm{~min}$ for each interval) centered at the times when measurements of $E_{z}$ switched to measurements of $B_{z}$ so that we get $E_{z}$ and $B_{z}$ data each for half interval. Then we assumed that the measurements of each component averaged over this interval are simultaneous.

The six components of $B$ and $E$ field were derived as follows: First, a sector was selected as the $+X^{\prime}$ aris of a temporary Cartesian coordinate system $\left(X^{\prime}, Y^{\prime}, Z^{\prime}\right.$, with $Z^{\prime}$ aligned with the $z$ antenna). The peak value of the $E$ field in the sector was taken as the amplitude of $E_{X^{\prime}}$, and wave phase of the $E$ field measured in this sector was taken as the reference phase. Then the $E_{Y \prime}$ component was obtained from the peak value and the relative phase shift (with respect to the $E_{X^{\prime}}$ ) measured in the sector perpendicular to the $X^{\prime}$ sector, while $E_{Z^{\prime}}$ was derived from the peak and phase data measured on the $z$ antenna. The components $E_{X^{\prime}}, E_{Y^{\prime}}$, and $E_{Z^{\prime}}$ were then rotated to the despun $S / C$ coordinates.

For example, if we choose sector 0 as the $+X^{\prime}$ direction, then

$$
\begin{gathered}
E_{X^{\prime}}=E_{x}(0) \cos (\omega t) \\
E_{Y^{\prime}}=E_{x}(2) \cos \left(\omega t+\varphi_{E_{x}(2)}-\varphi_{E_{z}(0)}\right) \\
E_{Z^{\prime}}=E_{z}(0) \cos \left(\omega t+\varphi_{E_{z}(0)}-\varphi_{E_{z}(0)}\right)
\end{gathered}
$$

where $E_{x}(i)$ and $E_{z}(i)$ represent the electric field (the peak value) measured on the $x$ and $z$ antennas, respectively, at the time when the antenna is in sector $i$.

Similarly, we have three components of $B$

$$
\begin{aligned}
& B_{X^{\prime}}=B_{y}(6) \cos \left(\omega t+\varphi_{B_{y}(6)}-\varphi_{E_{z}(0)}\right) \\
& B_{Y^{\prime}}=B_{y}(0) \cos \left(\omega t+\varphi_{B_{y}(0)}-\varphi_{E_{z}(0)}\right) \\
& B_{Z^{\prime}}=B_{z}(0) \cos \left(\omega t+\varphi_{B_{z}(0)}-\varphi_{E_{z}(0)}\right)
\end{aligned}
$$

where $B_{y}(i)$ and $B_{z}(i)$ are the magnetic field (the peak value) measured on the $y$ antenna and on the $z$ antenna, respectively, at the time when the $z$ antenna is in sector $i$. Knowing six components of $\mathbf{E}$ and $\mathbf{B}$, we may calculate the Poynting flux $S=\left(1 / \mu_{0}\right) E \times B$ in $X^{\prime}, Y^{\prime}$, and $Z$ ' coordinates, and then rotate the flux into the despun $S / C$ coordinates.

In general, any sector can be chosen as the reference sector $X^{\prime}$. In our calculation we chose sectors $6,7,0$, and 1, which are closer to the despun $X$ axis (see Figure 4), as reference sectors, and calculated the Poynting flux in the despun coordinates separately. The results from the four calculations were then averaged and shown (for 9.3-Hz channel) in Figure 5 as $S_{X}, S_{Y}$, and $S_{Z}$, with the standard deviation from the mean values overplotted. There were only nine switches of $B_{y} / B_{z}$ between 0900 and 1900. In the last panel of Figure 5 , the angle between the Poynting flux and the ambient magnetic field, which was measured as the average of the $B$ field over each $10 \mathrm{~min}$ period in the despun coordinates, is shown.

Figure 5 shows that near the equator, the angle between $S$ and $B$ was rather small $\left(\sim 20^{\circ}\right.$ near $\left.1600 \mathrm{UT}\right)$, which means the average wave energy flow was nearly parallel to the magnetic field. We note, however, that the standard deviation in $S_{X}$ and $S_{Y}$ for 1600 is large, which may suggest that the propagation of the waves was not in a unique direction. At high latitudes, at $\sim 1300$ UT, the Poynting flux was nearly perpendicular to the magnetic field. The intensity of the flux near the equator, $|\mathrm{S}| \sim 2.2 \times 10^{-9} \mathrm{~W} / \mathrm{m}^{2}$ at $1600 \mathrm{UT}$ is larger than that at higher latitudes $\left(\sim 1.1 \times 10^{-10} \mathrm{~W} / \mathrm{m}^{2}\right)$ at $\sim 1245$ UT.

\section{Wave Polarization}

In some cases the phase data may be used to derive the phase differences between field components to de- 

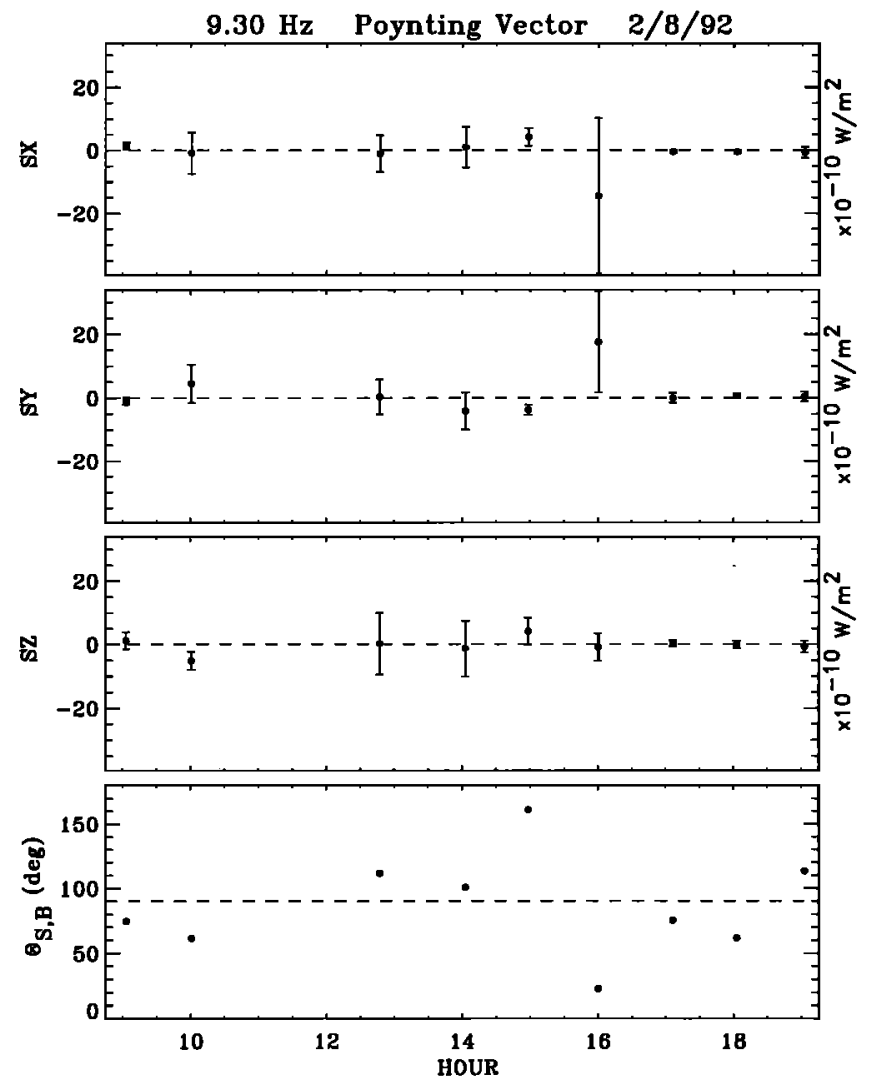

Fig. 5. Three components of the calculated Poynting flux $S_{X}, S_{Y}$, and $S_{Z}$, in the despun coordinates (the first three panels). The last panel is the angle between the Poynting flux and the magnetic field.

termine the polarization sense of the waves with respect to the ambient magnetic field. Near the closest aproach $\left(\Lambda \sim 30^{\circ}\right)$, the ambient magnetic field, about $(1072,418$, -2012) $\mathrm{nT}$ in the despun spacecraft coordinates $X, Y$, and $Z$ for the period 1155 to $1210 \mathrm{UT}$, was roughly in the $-Z$ direction $\left(\sim 30^{\circ}\right.$ tilted from the $Z$ axis). Thus the phase difference between signals in two perpendicular sectors (for example, $E_{x}(0)$ and $E_{x}(2), E_{x}(1)$ and $E_{x}(3)$, and so on) can be used to determine the polarization sense of the waves in the spin plane. Figure $6 a$ shows the phase shifts between $\mathbf{E}$ fields measured in two perpendicular sectors (denoted as $E_{Y^{\prime}}$ and $E_{X^{\prime}}$ ) at each highband frequency for the interval from 1155 to 1205 UT. There are eight pairs of $E_{Y}$ and $E_{X^{\prime}}$ at each frequency. Phase differences of $0^{\circ}$ to $180^{\circ}$ and $-180^{\circ}$ to $-360^{\circ}$ represent a right-handed polarization and is marked with $R$ on the right side of Figure $6 a$. The rest of the area is then for left-handed polarization and is marked with $L$. It is seen in Figure $6 a$ that the waves above $50 \mathrm{~Hz}$ appear to be right-hand polarized, which is consistent with the polarization of whistler mode waves. The polarization for the waves near and below $f_{c H^{+}}(35.5 \mathrm{~Hz})$, however, is uncertain with the dots scattered in both the $R$ and $L$ areas. As we mentioned earlier, during this interval the whistler mode signals extended to channel below $f_{\mathrm{cH}^{+}}$. The presence of both left- and right-hand polarization in these channels may imply that the signals are the mix of different wave modes. We also notice that some data points spread near the $0^{\circ}$ and $\pm 180^{\circ}$ lines, which correspond to linear polarization. Recently, Anderson et al $[1992 a, b]$ reported observations of electromagnetic ICWs in the Earth's outer magnetosphere that were linearly polarized at all magnetic latitudes they sampled (MLAT $< \pm 16^{\circ}$ ) in the region near dawn. These waves occurred at too high a frequency to have been affected by the crossover frequency and thus are suggested by the authors to be generated with linear polarization. It is possible that the approximately linearly polarized components seen in Figure 6a, which appear in channels below $f_{c H^{+}}$but higher than the crossover frequency $(\sim 10$ $\mathrm{Hz}$ in this case), are similar to those waves reported by Anderson et al. $[1992 a, b]$.

Near the equator, the ambient magnetic field, about $(-183,715,2.79) \mathrm{nT}$, is basically in the despun $+Y$ direction, $\sim 15^{\circ}$ away from the $+Y$ axis. In this case the centers of sectors $0,7,3$, and 4 lie near the plane perpendicular to the ambient magnetic field. Thus we may determine the polarization of the waves on the de-

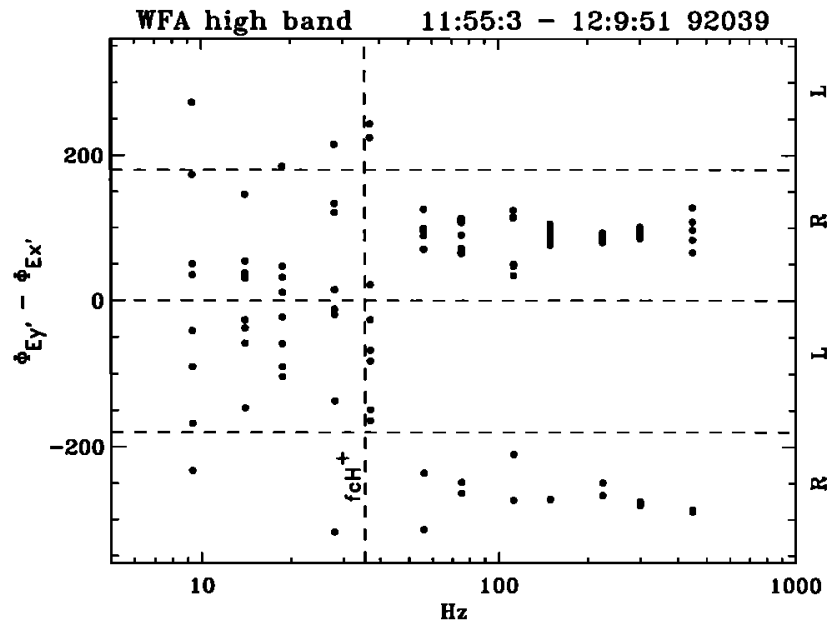

Fig. 6a. The phase difference between electric signals in two perpendicular sectors for the period 1155 to 1210 UT. Symbols $R$ and $L$ on the right side denote the right-handed and left-handed polarisation, respectively, implied by the phase shifts.

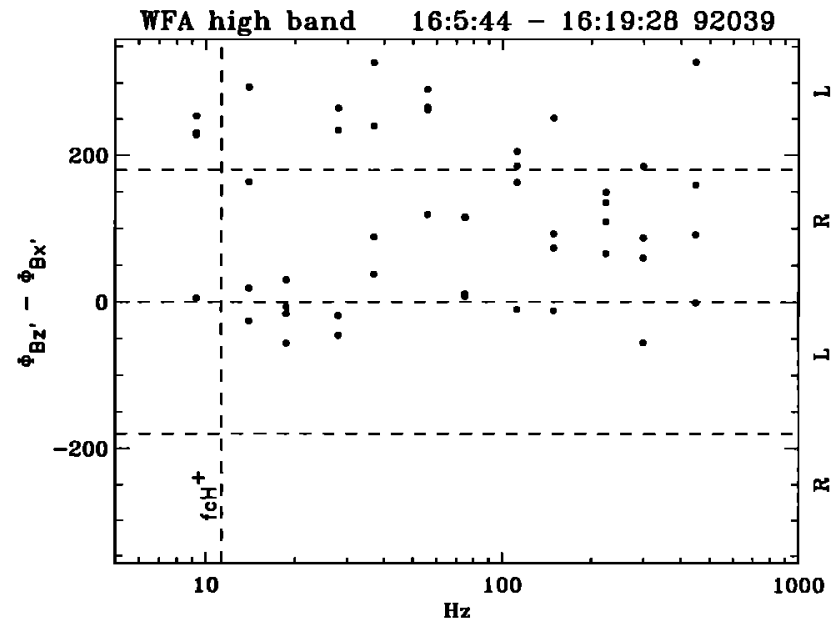

Fig. 6b. The phase difference between $B_{z}$ and $B_{y}$ for the period 1605 to 1620 UT. 
spun $X-Z$ plane by examining the phase shift between the signals in one of the four sectors and those on the $z$ antennas. The results for the period 1605 to 1620 UT are shown in Figure $6 b$. During this period the $z$ antenna was measuring magnetic signals. The plot shows the phase shifts between $B_{z}$ and $B_{y}$ measured in sectors $0,7,3$, or 4 . Although the results are more scattered than the previous ones, it can still be seen that the high-frequency channels (near and above 100 $\mathrm{Hz}$ ) exhibit right-handed polarization. Only the lowest channel $(9.3 \mathrm{~Hz})$ in this period falls below $f_{\mathrm{cH}^{+}}$. It appears to show a left-handed polarization. The magnetic measurements in the WFA are usually more noisy than the electric measurements. This may contribute to the larger scatter of the phase measurements seen in Figure $6 b$. For the frequencies near $50 \mathrm{~Hz}$ the data points scatter in both the $R$ and $L$ regions. We note that during this period the wave signals (especially the magnetic signals) at these frequencies have decreased to a very low level (see Figure 2b), thus the derivation of the polarization for these frequencies is less meaningful.

\section{Refractive Index}

In an attempt to better understand the observed wave modes we calculated the ratio $c B_{y} / E_{x}$ (where $c$ is the speed of light), which is an approximation of the refractive index $n$, and then compared the results with theoretically calculated values of $\pi$ using the cold plasma dispersion relation and the recently developed density model of the Io torus [Mei, 1992] (see also Mei et al. [1992]).

Figure $7 a$ show such a comparison for the equatorial period, $1605-1620 \mathrm{UT}(L \sim 7.7)$. The ratio $c B_{y} / E_{x}$ averaged over the period is plotted with the asterisks. For this period we assume that the wave normal is approximately parallel to the ambient magnetic field and calculate the dispersion relation of the cold plasma waves with $20^{\circ}$ wave angle. We used the general equation [Stix, $1962]$ to find the refractive index:

$$
A n^{4}-B n^{2}+C=0
$$

where

$$
\begin{gathered}
A=S \sin ^{2} \theta+P \cos ^{2} \theta \\
B=R L \sin ^{2} \theta+P S\left(1+\cos ^{2} \theta\right) \\
C=P R L
\end{gathered}
$$

and $S \equiv 0.5(R+L)$,

$$
\begin{gathered}
R \equiv 1-\sum_{k} \frac{\omega_{p k}^{2}}{\omega^{2}}\left(\frac{\omega}{\omega+\Omega_{k}}\right) \\
L \equiv 1-\sum_{k} \frac{\omega_{p k}^{2}}{\omega^{2}}\left(\frac{\omega}{\omega-\Omega_{k}}\right) \\
P \equiv 1-\sum_{k} \frac{\omega_{p k}^{2}}{\omega^{2}}
\end{gathered}
$$

where $\omega_{p k}$ is the plasma frequency of particle species $k$, while $\boldsymbol{\Omega}_{k}$ is the cyclotron frequency of species $k$, positive for ions and negative for electrons.

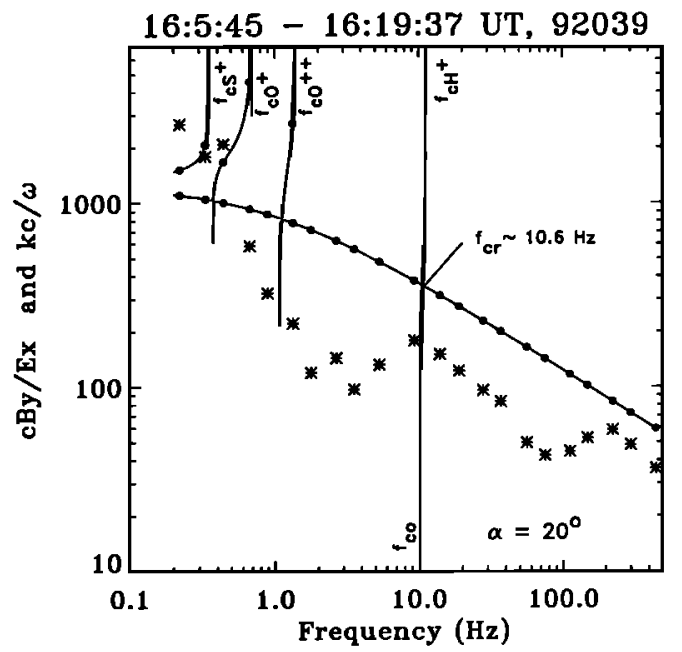

Fig. 7a. Ratio $c B_{y} / E_{x}$ (asterisks) and calculated refractive index (thin lines) assuming a $20^{\circ}$ wave angle for the interval 1605 to 1620 UT. The values of the refractive index at the WFA frequencies are marked with open circles (for $L$ mode) or solid circles (for $R$ mode).

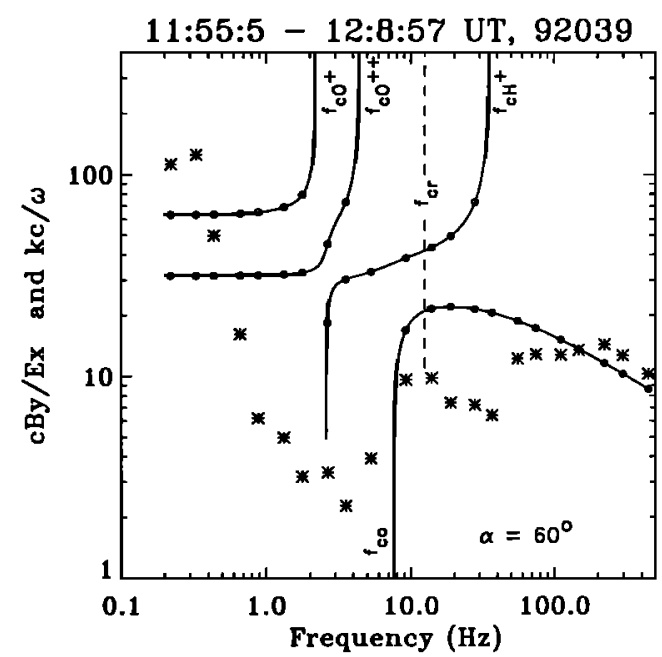

Fig. 7b. The same as Figure $7 a$, but for a $60^{\circ}$ wave angle and for 1155 to $1210 \mathrm{UT}$.

The electron density $\left(N_{e}\right)$ derived from the URAP observation is $\sim 400 \mathrm{~cm}^{-3}$ for this period [Stone, 1992b]. The ratios between the density of each ion species and $N_{e}$ are estimated using the model by $M e i$ [1992]. In the calculation we took $N_{e}, N_{\mathrm{H}^{+}}, N_{\mathrm{O}^{+}}, N_{\mathrm{O}^{++}}, N_{\mathrm{S}^{+}}$, and $N_{\mathrm{S}^{++}}$as $375,40,85,80,10$, and $40 \mathrm{~cm}^{-3}$, respectively. The calculated $n$ values are overplotted in Figure $7 a$ with thin lines. The more vertical lines represents the $L$ mode refractive index, while the more horizontal lines are for the $R$ mode. The calculated $n$ values at the center frequencies of the WFA channels are marked with solid circles (for the $R$ mode wave) or with open circles (for the $L$ mode wave).

The spectra for this period have shown a strong peak at $9.3 \mathrm{~Hz}$ (Figure 2b, central panels). This frequency is very close to the cutoff frequency, $f_{c o} \sim 10 \mathrm{~Hz}$, of a narrow $L$ mode branch which extends to the proton 
gyrofrequency (11.3 Hz). The lower part of this branch ( $<f_{c r}$ at $10.6 \mathrm{~Hz}$ ) is for the unguided $L$ mode wave. $\mathrm{Al}$ though the frequency resolution of the WFA instrument is too low to distinguish these characteristic frequencies $\left(f_{\mathrm{co}}, f_{\mathrm{cr}}\right.$, and $\left.f_{\mathrm{cH}}{ }^{+}\right)$in such a narrow frequency band $(10-11.3 \mathrm{~Hz})$, the observed $c B_{y} / E_{x}$ at $\sim 10 \mathrm{~Hz}$, which is closer to the values of the refractive index for the unguided $L$ mode wave may imply that the strong signal in the 9.3-Hs channel is the effect of nonconvective growth of unguided waves. The angle between the Poynting vector and the ambient magnetic field calculated near 1600 UT (Figure 5) is $\sim 22^{\circ}$. This seems to be consistent with the waves being unguided with a small wave angle, since for the unguided wave which has a closed refractive index surface, a quasi-parallel Poynting vector, which is in the same direction as the group velocity, implies a small wave angle [ef. Rauch and Rour, 1982]. However, as we will discuss later, a very large hot ion anisotropy is required to excite the ion cyclotron instability in this narrow frequency band. In the first few channels where a peak of the power density appeared in the $E_{x}$ spectra, the $n$ values are close to the two lowest $L$ mode branches.

The refractive index for the period of high latitudes between 1155 and $1210 \mathrm{UT}\left(L \sim 8.5, \Lambda \sim 30^{\circ}\right)$ is shown in Figure $7 b$ in the same format as that of Figure $7 a$. In the calculation of the dispersion relation we have used the following density numbers: $N_{e}=14, N_{\mathrm{H}^{+}}=$ 12.4, $N_{\mathrm{O}^{+}}=0.4$, and $N_{\mathrm{O}^{++}}=0.6 \mathrm{~cm}^{-3}$. The sulphur ions are neglected. We have calculated the refractive index for various wave angles and compared the results with the $c B_{y} / E_{z}$ obtained from the data. We found that at the frequencies of the first few channels of WFA the calculated values had better agreement with the data for the large wave angle than for the small wave angle. While for higher frequencies, not much improvement was seen by varying the wave angle. In Figure $7 b$ we show the calculations of the refractive index with $60^{\circ}$ wave angle.

The spectra for this period (Plate 1 and Figure 2a) show a peak at $9.3-\mathrm{Hz}$ channel seen in $B_{y}$, another peak above $f_{\mathrm{cO}}$ (in $2.67-\mathrm{Hz}$ channel), and a rather wide peak in the channels below $f_{c o}+(2.2 \mathrm{~Hz})$. The 9.3Hz channel falls in an $L$ mode branch between a cutoff frequency $f_{c o}=7.6 \mathrm{~Hz}$ and a crossover frequency at $\sim 12.5 \mathrm{~Hz}$. The measured $c B_{y} / E_{x}$ for this channel is closer to the $L$ mode branch although is smaller by a factor of 2 than the calculated $L$ mode $n$ value. The 2.67-Hz channel falls almost at the cutoff frequency (2.5 $\mathrm{Hz}$ ) of an $L$ mode band below $f_{c \mathrm{O}^{++}}$. The ratio $c B_{y} / E_{x}$ is much smaller than the calculated value for the central frequency but is very close to the $n$ near the cutoff frequency. It is possible that the channel has been affected by stronger signals near the cutoff frequency. The values of $c B_{y} / E_{x}$ for the first three channels are closer to the lowest branch of $L$ mode guided waves, but the observed values for the channels near $1 \mathrm{~Hz}$ are much lower than the calculated results. We note that in both Figure $7 a$ and $7 b$, most of the observed $c B_{y} / E_{x}$ values are lower than the corresponding calculated values. One of the possible reasons may be that the measured $E_{x}$ contains electrostatic components which should not be included in calculations of $c B_{y} / E_{x}$.

\section{Discussion}

Throughout the Io torus, Ulysses has observed intense ULF wave activity. The simultaneous observations of the waves in both magnetic and electric components below the proton gyrofrequency are convincing evidence of the existence of electromagnetic plasma waves. The frequency characteristics of these waves suggest that they are likely to be ion cyclotron noise which was excited by the resonant ions in the Io torus through the cyclotron instability.

Although the polarization analysis can only be conducted for the high-band channels of the WFA ( $>9$ Hz), the difference in polarisation between the waves below and above the local $f_{c H}+$ is clear, especially for the high-latitude period (Figure 6a). Above $f_{\mathrm{cH}^{+}}$, the waves exhibit right-hand polarization, and below $f_{c H^{+}}$, more data points fall in the $L$ mode region. The increase of randomness of polarization at the frequency below $f_{\mathrm{CH}^{+}}+$may imply that the observed waves have a rather wide range of wave angles or mixed wave modes. At the equator, only the lowest channel of the high-band data is below the local $f_{c H}+(11.3 \mathrm{~Hz})$, and the polarization in the channel is seen left-handed (Figure 6b).

The dominance of heavy ions near the equator in the Io torus produces broad frequency stop bands in which $L$ mode propagation is forbidden [Thorne and Moses, 1983; Mei et al., 1992]. This can also be seen from the numerical calculation results in Figure 7a. For example, no $L$ mode waves exist between $f_{\mathrm{co}++}$ and a cutoff frequency $\left(f_{c o}\right)$ at $\sim 10 \mathrm{~Hz}$. There is a narrow band for $L$ mode waves between the cutoff frequency and $f_{c H}+$. The waves below the crossover frequency $\left(f_{c r}\right)(\sim 10.6$ $\mathrm{Hz}$ ) in this $L$ mode branch are believed to be unguided waves [Mei, 1992], which are classified as class II waves by Rauch and Roux [1982], or as the $W$ branch following the notation by Horne and Thorne [1990]. The upper part of the branch (above $f_{c r}$ ) is possibly the $B$ waves described by Horne and Thorne's notation which are guided and could be very unstable, being excited by anisotropic resonant ions. The channel with a central frequency of $9.3 \mathrm{~Hz}$ detected strong signals near the equator (seen in $B_{y}$ in Plate 1 and Figure 2) and has a $c B_{y} / E_{x}$ ratio close to the refractive index of the $L$ mode $W$ branch at $\sim 10 \mathrm{~Hz}$. This seems to suggest that we may be seeing the unguided $L$ mode waves. However, there is an upper limit in frequency of ion cyclotron $L$ mode waves which closely depends on the anisotropy of the resonant protons [Kennel and Petschek, 1966]:

$$
f_{\max } / f_{c \mathrm{H}^{+}}=A^{+} /\left(A^{+}+1\right)
$$

where the anisotropy $A^{+}=T_{\perp} / T_{\|}-1$. Since the observed frequency is $9.3 \mathrm{~Hz}$ and $f_{\mathrm{cH}}+\sim 11.3 \mathrm{~Hz}$, the lower limit for $f_{\max } / f_{\mathrm{cH}}+$ is then $\sim 0.8$, which gives $A^{+}=4$ or $T_{\perp} / T_{\|}=5$. Such a large anisotropy seems unreasonable. Other mechanisms need to be explored to explain the observed equatorial waves. This is left for the future investigation.

At high latitudes (Figure $7 b$ ) there is a rather wide frequency band between $f_{C r}(\sim 12.5 \mathrm{~Hz})$ and $f_{r \mathrm{H}}+(35.4$ Hz) where the convective $L$ mode ICWs can propagate. However, the anisotropy $\mathrm{A}^{+}$will limit the waves to below a certain frequency. For example, taking a typi- 
cal $A^{+}=1$, the maximum wave frequency will be $1 / 2$ $f_{\mathrm{cH}}+$, which is $17 \mathrm{~Hz}$ for this peiod. As we have seen, a peak in this $L$ mode band appears at $\sim 10 \mathrm{~Hz}$, between $f_{c o}=7.63 \mathrm{~Hz}$ and $f_{c r}=12.5 \mathrm{~Hz}$, which belong to unguided $L$ mode. There is no Poynting vector calculation available for this period, but the Poynting flux calculated for this frequency near 1245 (Figure 5) when the spacecraft was still in about the same region show that the Poynting flux is quasi-perpendicular to the ambient magnetic field. This again seems to justify that the waves observed in this region may be very oblique, as we suggested in Figure $7 b$, since for the unguided wave, the quasi-perpendicular group velocity implies a large wave angle [Rauch and Roux, 1982]. The waves below $f_{\mathrm{O}^{+}}$and $f_{\mathrm{O}^{++}}$are seen in the spectra. Their peak channels fall within the frequency bands where ICWs propagate. Although the $c B_{y} / E_{x}$ ratio sometimes does not agree with the calculated refractive index as shown in the previous section, it is still reasonable to believe that these are the $L$ mode ICWs. Those waves could be easily excited in the equatorial region where the resonant energy is much lower and then propagate to high latitudes [Mei et al., 1992].

The wave amplitude required to produce strong pitch angle scattering loss of energetic ions can be estimated as [Thorne, 1983]

$$
E^{\prime} \sim 10^{2} E_{p}^{3 / 4}(\mathrm{keV}) L^{-7 / 2} \mathrm{mV} / \mathrm{m}
$$

where $E_{p}$ is the kinetic energy of the resonant protons. The resonance energy can be evaluated as $B^{2} / 8 \pi N$. Taking $B=738.6 \mathrm{nT}$ near the equator (for the period of 1605 to $1620 \mathrm{UT}$ ) and $N \sim 375 \mathrm{~cm}^{-3}$, we find the resonance energy to be $\sim 3.6 \mathrm{keV}$. This gives the required electric wave intensity for strong pitch angle scattering as $\sim 0.2 \mathrm{mV} / \mathrm{m}$. The observed ICWs power density of $E_{x}$ at channels 0.67 and $0.89 \mathrm{~Hz}$, which appear as peaks in the spectra, is $\sim 3 \times 10^{-6}(\mathrm{~V} / \mathrm{m})^{2} / \mathrm{Hz}$, which corresponds to an amplitude of $0.95 \mathrm{mV} / \mathrm{m}$, larger than the required amplitude.

At high latitudes (between 1155 to $1210 \mathrm{UT}$ ) the resonance energy was estimated to be $\sim 1 \mathrm{MeV}$ (taking $B=$ $2318 \mathrm{nT}$ and $N \sim 14 \mathrm{~cm}^{-3}$ ). For such energetic ions the required wave amplitude for strong diffusion is 10 $\mathrm{mV} / \mathrm{m}$. The observed wave power in the $0.67-\mathrm{Hz}$ channel which appears as a peak in the $E_{x}$ and $B_{y}$ spectra is $\sim 2.5 \times 10^{-4}(\mathrm{~V} / \mathrm{m})^{2} / \mathrm{Hz}$, corresponding to an amplitude of $\sim 8 \mathrm{mV} / \mathrm{m}$, close to the required amplitudes. Considering that the data for this estimation is the average power and the peak power is much higher, waves at this frequency may also contribute to the strong pitch angle scattering of energetic ions. It seems that the ICWs observed by Ulysses near the equator as well as at high latitudes $\left(\Lambda \sim 30^{\circ}\right)$ are strong enough to account for the pronounced depletion of energetic ions observed in the inner Jovian magnetosphere.

Acknowledgments. We would like to thank R. G. Stone, the principal investigator of the URAP experiment, for the opportunity to take part in this investigation. The authors would like to thanks F. Bagenal for kindly providing the equatorial plasme density data to produce the density contours in Figure 1. We are also grateful to Denise LengyelFrey of the University of Maryland for careful eramination of the WFA data. N.L. also thanks S. J. Monson and K. Goets of the University of Minnesota for their help in understanding the WFA data. N.L. and Y.M. would like to thank R. M. Thorne of UCLA for very helpful discussion. The work done at the University of Minnesota was supported by NASA under contract NAS5-31219.

The editor thanks R. B. Horne and J. A. Slavin for their assistance in evaluating this paper.

\section{REFERENCES}

Anderson, B. J., R. E. Erlandson, and L. J. Zanetti, A statistical study of Pc 1-2 magnetic pulsations in the equatorial magnetosphere, 1, Equatorial occurrence distributions, $J$. Geophys. Res., 97, 3075, 1992 a.

Anderson, B. J., R. E. Erlandson, and L. J. Zanetti, A statistical study of $\mathrm{Pc}$ 1-2 magnetic pulsations in the equatorial magnetosphere, 2, Wave properties, J. Geophys. Res., 97, $3089,1992 b$.

Bagenal, F., D. E. Shemansky, R. L. McNutt Jr., R. Schreier, and A. Eviatar, The abundence of $\mathrm{O}^{++}$in the Jovian magnetosphere, Geophys. Res. Lett., 19, 79, 1992.

Broadfoot, A. L., et al., Ertreme ultraviolet observations from Voyager 1 encounter with jupiter, Science, 204, 979,1979.

Farrell, W. M., et al., Ulysses observations of auroral hiss at high Jovian latitudes, Geophys. Res. Lett., in press, 1993.

Farrell, W. M., R. J. MacDowall, R. A. Hess, M. L. Kaiser, M. D. Desch, and R. G. Stone, An interpretation of the broadband VLF waves near the Io torus as observed by Ulysses, J. Geophys. Res., this issue.

Fraser, B. J., Pc 1-2 observations of heavy ion effects by synchronous satellite ATS-6, Planet. Space Sci., 30, 1229, 1982.

Fraser, B. J., J. C. Samson, Y. D. Hu, R. L. McPherron, and C. T. Russell, Electromagnetic ion cyclotron waves observed near the oxygen cyclotron frequency by ISEE 1 and 2, J. Geophys. Res., 97, 3063, 1992.

Gurnett, D. A., and C. K. Goertz, Ion-cyclotron waves in the Io plasma torus: Polarisation reversal of whistler mode noise, Geophys. Res. Lett., 10, 587, 1983.

Horne, R. B., and R. M. Thorne, Ion-cyclotron absorption at the second harmonic of the oxygen gyrofrequency, Geophys. Res. Lett., 17, 2225, 1990.

Kennel, C. F., and H. E. Petschek, Limit on stably trapped particle flures, J. Geophys. Res., 71, 1, 1966.

Krimigis, S. M., et al., Low-energy charged particle environment at Jupiter: A first look, Science, 204, 998, 1979.

Mauk, B. H., C. E. McIwain, and R. L. McPherron, Helium cyclotron resonance within the Earth's magnetosphere, Geophys. Res. Lett., 8, 103, 1981.

Mei, Y., The propagation characteristics and instability properties of low frequency electromagnetic waves in the Io plasma torus, $\mathrm{Ph} . \mathrm{D}$. thesis, Univ. of Calif., Los Angeles, 1992.

Mei, Y., R. M. Thorne, and R. B. Horne, Ion-cyclotron waves at Jupiter: Possibility of detection by Ulysses, Geophys. Res. Lett., 19, 629, 1992.

Roux, A., S. Perraut, J. L. Rauch, C. de Villedary, G. Kremser, A. Korth, and D. T. Young, Wave-particle interactions near $\boldsymbol{\Omega}_{\mathrm{He}^{+}}$observed on board GEOS 1 and 2, 2, Generation of ion cyclotron waves and heating of $\mathrm{He}^{+}$ ions, J. Geophys. Res., 87, 8174, 1982.

Rauch, J. L., and A. Roux, Ray tracing of ULF waves in a multicomponent magnetospheric plasma: Consequencies for the generation mechanism of ion cyclotron waves, $J$. Geophys. Res., 87, 8191, 1982.

Stix, T. H., The Theory of Plasma Waves, McGraw-Hill, New York, 1962.

Stone, R. G., et al., The unified radio and plasma wave investigation, Astron. Astrophys. Supp. Ser., 92, 291, $1992 a$.

Stone, R. G., et al., Ulysses radio and plasma wave obser- 
vations in the Jupiter environment, Science, 257, 1524, $1992 b$.

Thorne, R. M., Microscopic plasma processes in the Jovian magnetosphere, in Physics of the Jovian Magnetosphere, edited by A. J. Dessler, p. 454, Cambridge University Press, New York, 1983.

Thorne, R. M., and J. J. Moses, Electromagnetic ion-cyclotron instability in the multi-ion Jovian magnetosphere, Geophys. Res. Lett., 10, 631, 1983.

Thorne, R. M., and J. J. Moses, Resonant instability near the two-ion crossover frequency in the Io plasma torus, $J$. Geophys. Res., 90, 6311, 1985.

Thorne, R. M., and F. L. Scarf, Voyager 1 evidence for ioncyclotron instability in the vicinity of the Io plasma torus, Geophys. Res. Lett., 11, 263, 1984.

Young, D. T., S. Perraut, A. Rour, C. de Villedary, R. Gendrin, A. Korth, G. Kremser, and D. Jones, Wave-particle interaction near $\boldsymbol{\Omega}_{\mathrm{He}^{+}}$observed on GEOS 1 and 2,1 ,
Propagation of ion cyclotron waves in $\mathrm{He}^{+}-$rich plasma, J. Geophys. Res., 86, 6755, 1981.

A. Balogh and R. J. Forsyth, Imperial College of Science and Technology, London, United Kingdom.

P.Canu, N.Cornilleau-Wehrlin, C.de Villedary, and L.Rezeau, Centre de Recherche en Physique de l'Environnement, Centre National d'Etudes des Télécommunications, 92131 Issy les Moulineaur, France.

P. J. Kellogg and N. Lin, School of Physics and Astronomy, University of Minnesota, Minneapolis, MN 55455.

R. J. MacDowall, NASA Goddard Space Flight Center, Greenbelt, MD 20771.

Y. Mei, Department of Atmospheric Sciences, University of California, Los Angeles, 90024.

(Received April 12, 1993; revised July 29, 1993; accepted August 31, 1993.) 